\title{
Phlebologie
}

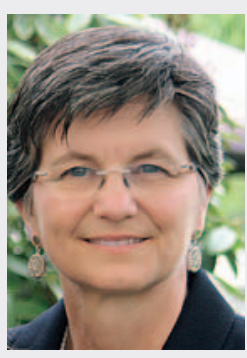

Erika Mendoza

\section{Liebe Leserinnen, liebe Leser der Phlebologie,}

ein bemerkenswertes Jahr geht zu Ende, in dem wir uns alle unvorhergesehen neuen Themen öffnen, Abläufe organisieren, privat und beruflich Einbußen und Umstellungen hinnehmen mussten. Sicherlich geht es im kommenden Jahr ähnlich weiter - eine Zäsur, die uns innehalten und nachdenken lässt, denn „immer weiter so“, schneller, besser, effizienter, das hat uns COVID gezeigt, darauf können wir uns nicht verlassen. Eine Besinnung auf Grundlegendes scheint gerade jetzt wichtig. Somit passt das Thema „Anatomie“ als Grundpfeiler der Medizin im Allgemeinen und in der Phlebologie im Besonderen gut in diese Zeit.

Mit diesem Heft halten Sie das erste aus einer Doublette zum Thema Anatomie mit Blick auf die Diagnostik und Therapie bei phlebologischen Erkrankungen in der Hand. Als ich die Autoren anfragte, konnte ich mir nicht vorstellen, was für eine hervorragende Resonanz ich bei ihnen erhielt. Noch mehr erfreute mich die Begeisterung, mit der sie ihre Beiträge zu einem sonst doch so „trockenen“ Thema wie der Anatomie verfassten.

In diesem ersten Heft geht es um die Anatomie der tiefen Bein- und Beckenvenen sowie der Lymphbahnen als zentraler Organe der Phlebologie und um alle begleitenden Strukturen wie Lymphknoten, Nerven und Arterien. Das zweite Heft handelt von der Anatomie der oberflächlichen Beinvenen mit ihren Verbindungen zum pelvinen und zum tiefen Venensystem.

Die Autoren haben die Anatomie mit der Diagnostik verknüpft, dabei praktische Tipps zur besseren Veranschaulichung der beschriebenen Strukturen im Ultraschall gegeben. Außerdem wird auf die klinischen Implikationen eingegangen; somit ist dieses „alte Thema“ absolut zeitgemäß aufgearbeitet und extrem informativ, sowohl für die „alten Hasen“ unter uns als auch für jeden, der sich neu mit dem Thema Phlebologie auseinandersetzt. Ich wünsche dem Leser dieselbe Freude beim Entdecken von Altbekanntem in neuem Kleid - aber auch von neuen klinisch relevanten Informationen.

Und für die bevorstehende Weihnachtszeit sowie den Jahreswechsel wünsche ich Ihnen viel Gesundheit, Zeit für das Wesentliche und weiterhin viel Freude an unserem/-r wunderschönen Beruf(ung), der Phlebologie.

Ihre

Erika Mendoza, Wunstorf 